\title{
New and emerging HDAC inhibitors for cancer treatment
}

\author{
Alison C. West ${ }^{1}$ and Ricky W. Johnstone ${ }^{1,2}$ \\ ${ }^{1}$ Cancer Therapeutics Program, The Peter MacCallum Cancer Centre, East Melbourne, Victoria, Australia. \\ ${ }^{2}$ Sir Peter MacCallum Department of Oncology, University of Melbourne, Parkville, Victoria, Australia.
}

\begin{abstract}
Epigenetic enzymes are often dysregulated in human tumors through mutation, altered expression, or inappropriate recruitment to certain loci. The identification of these enzymes and their partner proteins has driven the rapid development of small-molecule inhibitors that target the cancer epigenome. Herein, we discuss the influence of aberrantly regulated histone deacetylases (HDACs) in tumorigenesis. We examine HDAC inhibitors (HDACis) targeting class I, II, and IV HDACs that are currently under development for use as anticancer agents following the FDA approval of two HDACis, vorinostat and romidepsin.
\end{abstract}

\section{Introduction}

Histone acetylation is an important determinant of gene expression. Acetylation is generally associated with elevated transcription, while deacetylated histones are often associated with gene repression. Histone deacetylases (HDACs) are critical regulators of gene expression that enzymatically remove the acetyl group from histones. A broader role for HDACs as acyl-lysine "erasers" is also emerging with the discovery of additional acyl-lysine histone modifications such as crotonylation, succinylation, and malonylation $(1,2)$. The activity of HDACs on nonhistone proteins is also a key aspect of HDAC function (3). However, while a large number of nonhistone HDAC substrates have been identified, the molecular and biological consequences of nonhistone protein deacetylation is yet to be elucidated for the majority of these targets. Three classical HDAC classes (I, II, and IV) containing 11 HDACs have been identified thus far and are classified according to their homology to yeast proteins, subcellular location, and enzymatic activities (these distinctions are further described in Table 1 and Figure 1). The class III HDACs, or sirtuins, possess NAD-dependent catalytic sites and have some overlapping functions with the classical HDACs. However, sirtuins are not hindered by conventional HDAC inhibitors (HDACis), and as such will not be discussed herein. The biological outcome of HDAC inhibition is dependent on the HDAC specificity of the compound and intrinsic operation of cell-signaling pathways. HDACis are best characterized as anticancer agents, and investigation into their mechanisms of action and clinical efficacy remains robust and active, and will be discussed further.

\section{Rationale for targeting HDACs in cancer}

An early indication that genome-wide changes in histone acetylation may underpin cancer onset and progression came from a study demonstrating a global loss of monoacetylation and trimethylation on histone H4 in cancer cells (4). Numerous correlative studies have demonstrated aberrant expression of HDACs in human tumors (Table 1), and expression of HDAC1, -5 , and -7 can serve as a molecular biomarker of tumor versus normal tissue (5). Interestingly, in several cancer types, such as prostate (6), colorectal (7), breast (8), lung $(9,10)$, liver (11), and gastric cancer (12), overexpression of individual HDACs correlated with

Conflict of interest: The Johnstone laboratory receives grant support from Novartis. Citation for this article: J Clin Invest. 2014;124(1):30-39. doi:10.1172/JCI69738. significant decreases in both disease-free and overall survival and was able to predict poor patient prognosis independent of other variables such as tumor type and disease progression. Indeed, the overexpression of HDACs has been linked to key events of tumorigenesis such as the epigenetic repression of the tumor suppressor gene CDKN1A (encoding the cyclin-dependent kinase inhibitor p21 waf1/cip1; ref. 13) and key genes encoding DNA damage repair enzymes such as breast cancer 1, early onset (BRCA1) and ataxia telangiectasia and Rad3 related (ATR) (14). However, it is clear that HDAC overexpression is not always a negative prognostic marker; indeed, elevated HDAC6 levels predicted better prognosis in patients with estrogen receptor-positive (ER-positive) breast cancer (15). Furthermore, despite potent clinical activity of panHDACis in cutaneous T cell lymphoma (CTCL), the overexpression of HDAC6 correlated with improved prognosis, and acetylated H4 was associated with more aggressive lesions (16). Thus, the level of HDAC expression and/or histone acetylation may not necessarily indicate clinical sensitivity to oncology drugs, including HDACis.

Aberrant activity of HDACs is, however, linked to key oncogenic events. The genetic knockdown of individual HDACs, most notably HDAC1, $-2,-3$, and -6 , in a variety of tumor types such as colon, breast, and lung and acute promyelocytic leukemia (APL) induced apoptosis and cell cycle arrest (further described in Table 1), implicating HDAC activity as a key mediator of survival and tumorigenic capacity in these settings. Direct deacetylation of the tumor suppressor p53 by HDACs leads to decreased transcriptional activity (17), and the upregulation of oncogenes such as BCL2 is induced by HDAC-mediated deacetylation and activation of the transcription factors SP1 and C/EBP $(18)$. The aberrant recruitment of HDACs to certain gene loci through binding to oncogenic fusion proteins has also been proposed as an important mechanism of tumorigenesis. For example, AML1-ETO (a fusion of the acute myeloid leukemia 1 [AML1] and eight twenty-one [ETO] proteins resulting from $t[8 ; 21]$ ) and PML-RAR $\alpha$ (a fusion of promyelocytic leukemia [PML] and retinoic acid receptor $\alpha$ [RAR $\alpha]$ fusion arising from $t[15 ; 17])$ gain corepressor activity upon aberrant recruitment of HDAC complexes (containing nuclear receptor corepressor [NCoR; ref. 19]) and gain subsequent leukemogenic potential upon aberrant recruitment of $\mathrm{mSin} 3 \mathrm{a}$ and silencing mediator for retinoid and thyroid receptors (SMRT) (20). Indeed, HDACis are potent inducers of apoptosis and terminal differentiation in leukemias expressing AML1-ETO and PML-RAR $\alpha(21,22)$. 
Table 1

Aberrant regulation of HDACs in cancer

\begin{tabular}{|c|c|c|c|c|}
\hline HDAC & $\begin{array}{l}\text { Normal and oncogenic } \\
\text { protein associations }\end{array}$ & Expression in cancer & Genetic evidence & Reference \\
\hline \multicolumn{5}{|c|}{ Class I (homologous to RDP3 yeast protein, nuclear location, ubiquitous tissue expression) } \\
\hline HDAC1 & $\begin{array}{l}\text { HDAC2, CoREST, NuRD, } \\
\text { Sin3, AML1-ET0, PML, } \\
\text { PLZF, BCL6, p53, } \\
\text { AR, ER, Rb/E2F1 }\end{array}$ & $\begin{array}{l}\text { Elevated in gastric }{ }^{A} \text {, } \\
\text { breast }^{B} \text {, colorectal, } \\
\mathrm{HL} \text {, lung }{ }^{\mathrm{A}} \text {, liver }{ }^{\mathrm{A}}\end{array}$ & $\begin{array}{l}\text { KD induced growth arrest, decreased viability, and } \\
\text { increased apoptosis in colon, breast, and osteosarcoma } \\
\text { cancer cells and increased survival of mice with overt } \\
\text { PML-RAR } \alpha \text {-mediated APL; HDAC1 KO/HDAC2 KD } \\
\text { induced growth arrest in fibroblasts; KO induced } \\
\text { genomic instability and arrest and reduced survival } \\
\text { of transformed cells in vivo }\end{array}$ & $\begin{array}{c}7-9,11,12 \\
95-106\end{array}$ \\
\hline HDAC2 & $\begin{array}{l}\text { HDAC1, CoREST, NuRD, } \\
\text { Sin3, AML1-ET0, } \\
\text { PML, PLZF, Bcl6 }\end{array}$ & $\begin{array}{l}\text { Elevated in gastric }{ }^{\mathrm{A}} \text {, } \\
\text { prostate }^{\mathrm{A}} \text {, } \text { colorectal }^{\mathrm{A}} \text {, } \\
\text { HL, CTCL }\end{array}$ & $\begin{array}{l}\text { KD induced growth arrest, decreased viability, and } \\
\text { increased apoptosis in colon and breast cancer cells } \\
\text { and induced apoptosis and decreased lung cancer in } \\
\text { vivo; HDAC1 KO/HDAC2 KD induced growth arrest in } \\
\text { fibroblasts; KO induced genomic instability and arrest } \\
\text { and reduced survival of transformed cells in vivo; } \\
\text { KD induced apoptosis and decreased lung cancer in vivo }\end{array}$ & $\begin{array}{c}6,7,12 \\
96-101,103 \\
106-109\end{array}$ \\
\hline HDAC3 & $\begin{array}{l}\text { HDAC4, HDAC5, HDAC7, } \\
\text { NCoR/SMRT, AML1-ETO, } \\
\text { PML, PLZF, PML-RAR } \alpha \text {, } \\
\text { PLZF-RAR } \alpha, \text { Bcl6, } \\
\text { STAT1, STAT3, GATA1, } \\
\text { GATA2, NF-кB }\end{array}$ & $\begin{array}{l}\text { Elevated in gastric }{ }^{A} \text {, } \\
\text { breast }^{A B}, A L L \text {, colorectal, } \\
\text { HL; decreased in liver }\end{array}$ & $\begin{array}{l}\mathrm{KD} \text { in colon cancer cells decreased survival, increased } \\
\text { apoptosis, and relieved transcriptional repression } \\
\text { mediated by PML-RAR } \alpha \text { in APL cells }\end{array}$ & $\begin{array}{c}7,8,12,25 \\
78,96,97 \\
99,100,105 \\
110-113\end{array}$ \\
\hline HDAC8 & & Elevated in neuroblastoma & $\begin{array}{l}\text { KD reduced proliferation of lung, colon, } \\
\text { and cervical cancer cells }\end{array}$ & 50,71 \\
\hline \multicolumn{5}{|c|}{ Class Ila (homologous to Hda1 yeast protein, shuttle between nucleus and cytoplasm, tissue-restricted expression) } \\
\hline HDAC4 & HDAC3-NCoR, GATA1 & & $\begin{array}{l}\text { KD in chondrosarcoma cells increased VEGF } \\
\text { expression and reduced growth and induced apoptosis } \\
\text { of colon and glioblastoma tumors in vivo }\end{array}$ & $\begin{array}{c}76-78 \\
105,114\end{array}$ \\
\hline HDAC5 & $\begin{array}{l}\text { HDAC3-NCoR, GATA1, } \\
\text { GATA2 }\end{array}$ & $\begin{array}{l}\text { Elevated in medulloblastoma; } \\
\text { decreased in lung }\end{array}$ & $\begin{array}{l}\text { KD decreased medullablastoma cell growth } \\
\text { and viability }\end{array}$ & $\begin{array}{c}9,78 \\
105,115\end{array}$ \\
\hline HDAC7 & HDAC3-NCoR, ER $\alpha$ & $\begin{array}{l}\text { Elevated in ALL; decreased } \\
\text { in lung }\end{array}$ & $\begin{array}{l}\text { KD induced growth arrest in colon } \\
\text { and breast cancer cells }\end{array}$ & $\begin{array}{c}10,78 \\
110,116\end{array}$ \\
\hline HDAC9 & & $\begin{array}{l}\text { Elevated in ALL, } \\
\text { medullablastoma }\end{array}$ & $\begin{array}{l}\text { KD of HDAC9/10 inhibited homologous } \\
\text { recombination and increased sensitivity to DNA } \\
\text { damage and decreased medullablastoma } \\
\text { cell growth and viability }\end{array}$ & $\begin{array}{l}110,115 \\
117\end{array}$ \\
\hline \multicolumn{5}{|c|}{ Class Ilb (homologous to yeast protein Hda1, mostly cytoplasmic location, tissue-restricted expression) } \\
\hline HDAC6 & $\begin{array}{l}\alpha \text {-Tubulin, HSP90, } \\
\text { HDAC11 }\end{array}$ & $\begin{array}{l}\text { Elevated in breast }{ }^{\mathrm{B}}, \mathrm{CTCL} \\
\text { decreased in lung }\end{array}$ & $\begin{array}{l}\text { KD decreased VEGF expression and decreased } \\
\text { cell viability due to accumulation } \\
\text { of misfolded proteins }\end{array}$ & $\begin{array}{c}10,16,83 \\
105,118,119\end{array}$ \\
\hline HDAC10 & & & $\begin{array}{l}\text { KD of HDAC9/10 inhibited homologous } \\
\text { recombination and increased sensitivity to DNA } \\
\text { damage and decreased VEGF expression }\end{array}$ & 117,119 \\
\hline \multicolumn{5}{|c|}{ Class IV (unknown yeast protein homology, cytoplasmic location, tissue-restricted expression) } \\
\hline Class 11 & HDAC6 & $\begin{array}{l}\text { Elevated in breast, renal, } \\
\text { liver }\end{array}$ & $\begin{array}{l}\text { KD induced apoptosis in colon, prostate, } \\
\text { breast, and ovarian cancer lines }\end{array}$ & $\begin{array}{c}94,120 \\
121\end{array}$ \\
\hline
\end{tabular}

Alndependent prognosis indicator. ${ }^{B}$ Associated with enhanced prognosis. KD, knockdown; HL, Hodgkin lymphoma; ALL, acute lymphoblastic leukemia.

Intriguingly, downregulation and deletion of HDACs may play a role in cancer progression. HDAC4 is routinely decreased in gastric tumors, HDAC1 somatic mutations were detected in approximately $8 \%$ of dedifferentiated human liposarcomas (23), and a frame-shift mutation causing dysfunctional HDAC2 expression was observed in human epithelial cancers with microsatellite instability (24). Together these data suggest a putative tumor suppressor role for certain HDACs. Indeed, recent studies by our laboratory and others using HDAC knockout and knockdown technologies provide strong functional evidence supporting tumor suppressor roles for HDAC1, -2, and -3 (25-27). Genetic knockout of Hdac1 and Hdac2 enhanced spontaneous tumorigenesis in a 


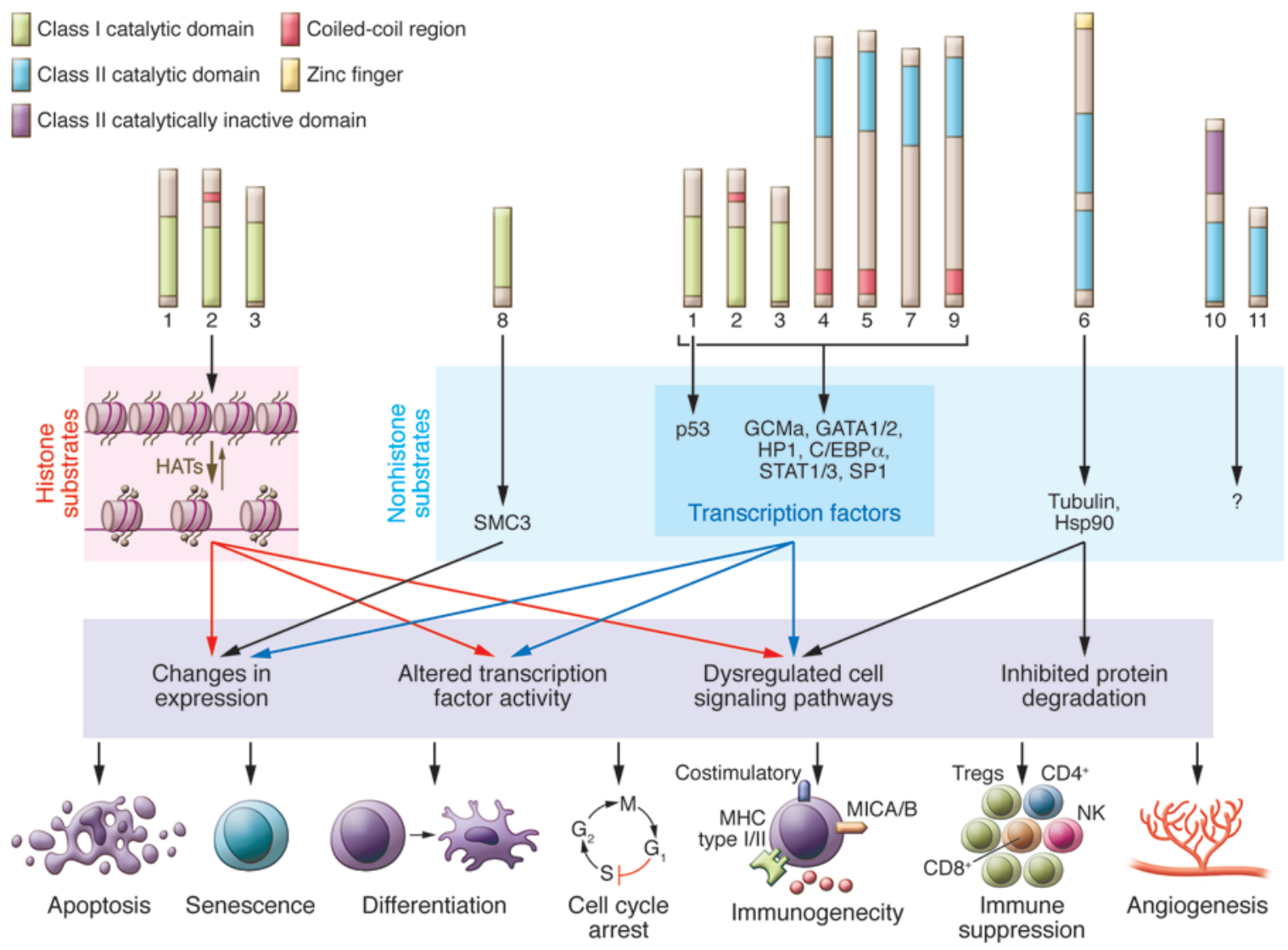

\section{Figure 1}

The molecular targets of HDACs, downstream cellular pathways, and anticancer outcomes of HDAC inhibition. HDAC substrates include histones and nonhistone proteins. Histones are the primary substrates for HDAC1, -2 , and -3 , while other cellular proteins are targeted by one or more class I and class II HDACs. Some known HDAC targets, downstream molecular changes that occur following HDAC inhibition, and associated biological pathways that mediate antitumor responses are shown. The HDACs, substrates, and molecular responses are color matched to illustrate functional relationships. The best-characterized biological consequences of HDACi treatment of tumor cells are shown on the lowest tier of the diagram. SMC3, structural maintenance of chromosomes 3; GCMa, glial cells missing homolog 1; HAT, histone acetyltransferase; HP1, heterochromatin protein 1.

dose-dependent manner by relieving a p53-dependent oncogenic barrier (26), and HDAC1 genetic and pharmacologic inhibition accelerated tumorigenesis of precancerous cells harboring oncogenic lesions (27). Bhaskara et al. observed that downregulation of the HDAC3-containing complex NCoR was apparent in approximately $30 \%$ of human hepatocellular carcinomas (HCCs), and the tumor suppressor function of HDAC3 was confirmed when liver-specific HDAC3 knockdown resulted in overt HCC (25). HDACs may also suppress the development of metastasis, as HDACi treatment alleviated repression of metastasis-related genes, subsequently promoting the migration of human tumor cell lines in vitro $(28,29)$ and enhancing metastasis in vivo $(28)$. It will be important to elucidate the impact of these class I HDACs at each step of tumorigenesis in order to explain their apparent paradoxical roles as tumor suppressors in developing tumors and as therapeutic targets in established neoplasms. HDACis are currently being studied in clinical trials for use against non-cancer diseases such as rheumatoid arthritis (30). Based on the limited experimental data available to date, one might predict that patients chronically treated with HDACis may be at risk of developing therapy-induced tumors. However, there is no clinical data as yet demonstrating the development of malignancies following HDACi treatment of patients with noncancerous diseases.

\section{HDACis as anticancer agents}

A vast array of both natural and synthetic chemical compounds function as HDACis. Two HDACis, vorinostat and romidepsin, are FDA approved for use against refractory CTCL, and many others are currently being clinically assessed (see Table 2 for a list of recent clinical trials assessing HDACi efficacy against cancer). HDACis are classified into groups based on their chemical structure, including hydroxamic acids (trichostatin A, vorinostat), carboxylic acids (valproate, butyrate), aminobenzamides (entinostat, mocetinostat), cyclic peptides (apicidin, romidepsin), epoxyketones (trapoxins), and hybrid molecules. There are 11 class I, II, and IV HDACs, and the target specificity of HDACis and the requirement for specific and selective inhibition of HDACs to achieve therapeutic efficacy remain topics of debate. Traditionally, tests examining the bioactivity of HDACis in vivo have included assessment of histone acetylation levels by Western blot or immunohistochemistry, and more recently, fluorescence-based reporter assays examining HDAC activity in the peripheral white 
Table 2

HDACis in current clinical trials

\begin{tabular}{|c|c|c|c|c|}
\hline HDACi & ghest phase trial & Cancer type & Best clinical outcome & Reference \\
\hline Panobinostat (LBH-589) & III & CTCL & $\begin{array}{c}\text { Ongoing; promising in phase II against } \mathrm{CTCL} \\
\text { (74\% tumor reduction), } \mathrm{HL}(74 \% \text { tumor reduction and } \\
27 \% \text { OR), and WM (MR or better in } 47 \% \text { of patients, } 50 \% \text { SD) }\end{array}$ & $122-124$ \\
\hline Belinostat (PXD101) & II & Thymoma & Significantly enhanced survival & 125 \\
\hline Entinostat (MS275) & II & Melanoma & Some clinical activity, promising PK and PD values & $126-128$ \\
\hline Mocetinostat (MGCD01030) & II & B cell malignancies & Disease control (35\% rate) in $\mathrm{HL}$ & 129 \\
\hline Givinostat (ITF2357) & m) & $\begin{array}{l}\text { JAK2 } 2617 F-e x p r e s s i n g \\
\text { myeloproliferative neoplasms }\end{array}$ & $\begin{array}{l}\text { Pruritus relief }(\sim 100 \%) \text {, splenomegaly reduction } \\
\text { (75\% of PV/ET and } 38 \% \text { of MF patients) }\end{array}$ & 130 \\
\hline Practinostat (SB939) & II & Prostate cancer & Limited clinical efficacy to date but promising PK values & 131,132 \\
\hline Chidamide (CS055/HBI-8000) & II & Solid tumors and lymphomas & Ongoing; PR was observed in 5/31 patients during phase I & 61 \\
\hline Quisinostat (JNJ-26481585) & ॥ & CTCL & $\begin{array}{c}\text { Ongoing; } 31.6 \% \text { reduction in mSWAT score } \\
\text { of tumor burden, } 1 / 19 \mathrm{CR}, 4 / 19 \mathrm{PR}\end{array}$ & 63 \\
\hline Abexinostat (PCl- 24781) & II & $\mathrm{FL}$ & Tumor reduction in $86 \%$, ORR of $64 \%$ & 64 \\
\hline CHR-3996 & Va & /arious (mostly solid tumors) & Favorable PK and PD values & 60 \\
\hline AR-42 & 1 & Hematological malignancies & $\begin{array}{l}\text { Ongoing, minor clinical responses in myeloma } \\
\text { and T cell lymphoma }\end{array}$ & 62 \\
\hline
\end{tabular}

CR, complete response; ET, essential thrombocythemia; FL, follicular lymphoma; MF, myelofibrosis; MR, minimal response; OR, overall response; ORR, overall response rate; PD, pharmacodynamic; PK, pharmacokinetic; PR, partial response; PV, polycythaemia vera; SD, stable disease; WM, Waldenström macroglobulinemia.

blood cells of HDACi-treated cancer patients have been developed (31). These non-HDAC class-selective bioassays might prove useful for determining HDACi pharmacodynamics in clinical trials. However, reliable determination of HDACi target specificity in vitro using standard assays with recombinant HDAC proteins has been hindered due to protein misfolding, lack of enzymatic activity, and most importantly, the inappropriate assessment of isolated HDACs that exist as multiprotein complexes in physiologic conditions. Furthermore, the substrate specificity of HDACs, in particular class IIa HDACs, is yet to be fully elucidated, which means that the precise surrogate biomarkers of individual HDAC inhibition are also not defined. Importantly, the activity of class I HDACs increases upon complex formation $(32,33)$, and recent pivotal studies using sophisticated chemoproteomics and chemical phylogenetic approaches demonstrated that commonly used HDACis have a higher degree of specificity than originally proposed $(34,35)$. HDACis can be used as "bait" to explicitly discriminate between multiprotein complexes containing the same HDAC target (34). Interestingly, class IIa HDACs were rarely identified as direct targets of HDACis, possibly due to their low catalytic activity, suggesting a role for this class in the recognition of and binding to acetylated histones (histone "reading") with subsequent recruitment of additional epigenetic modifiers, as opposed to active deacetylation $(34,35)$. Corroborating these findings in vivo remains a further challenge, but the development of the HDAC chemical phylogenetic tree (35) and identification of specific substrates of individual HDACs, such as SMC3 for HDAC8 (36), may lead to more focused in vivo investigations into HDAC and HDACi selectivity and specificity.

Studies utilizing genetic knockdown or knockout of HDACs have provided clues as to the most important HDACs to target in cancer. Depletion of HDAC1, $-2,-3,-4$, or -11 was sufficient to induce apoptosis in a range of tumor cells lines, whereas HDAC1, -2 , and -4 were required to maintain cancer cell survival in vivo (specific experiments are listed in Table 1). It will be crucial to determine whether the antitumor effects observed upon knockout or knockdown of HDACs listed in Table 1 was due to lack of HDAC activity specifically or was linked to disruption of HDACcontaining complexes that might have epigenetic or other regulatory activities. This distinction is particularly relevant to HDAC1, -2 , and -3 , which are key catalytic subunits of various complexes and are recruited by oncogenic fusion proteins (protein associations of individual HDACs are listed in Table 1 and illustrated in Figure 2). Furthermore, the pharmacologic inhibition of HDACs will almost certainly not be equivalent to genetic knockdown/ knockout (37), especially in terms of effects on multiprotein complex formation and function, and for less catalytically active HDACs such as those in class IIa. Additional investigations will likely result in the identification of the key HDAC-containing complexes required by tumor cells for survival, the specific functions of HDACs within these complexes, and the molecular and biological consequences of pharmacologic inhibition of these HDACs. These findings will hopefully allow for a more rational approach to stratifying patients for HDACi treatment. Furthermore, it may be possible to investigate the correlation between inhibiting specific HDAC-dependent complexes in particular tumor types with clinical outcomes following HDACi treatment, allowing for the identification of novel HDACi response biomarkers.

\section{HDACi mechanisms of action}

HDACis can induce tumor cell apoptosis, growth arrest, senescence, differentiation, and immunogenicity, and inhibit angiogenesis, as depicted in Figure 1. It is almost impossible to conceive that a single mechanism of action will be identified that is responsible for antitumor effects across different tumor types mediated by HDACis with diverse specificity profiles. Almost certainly, the biological effects and therapeutic outcomes will depend on the genetic lesions driving the tumor of interest and the HDACi 


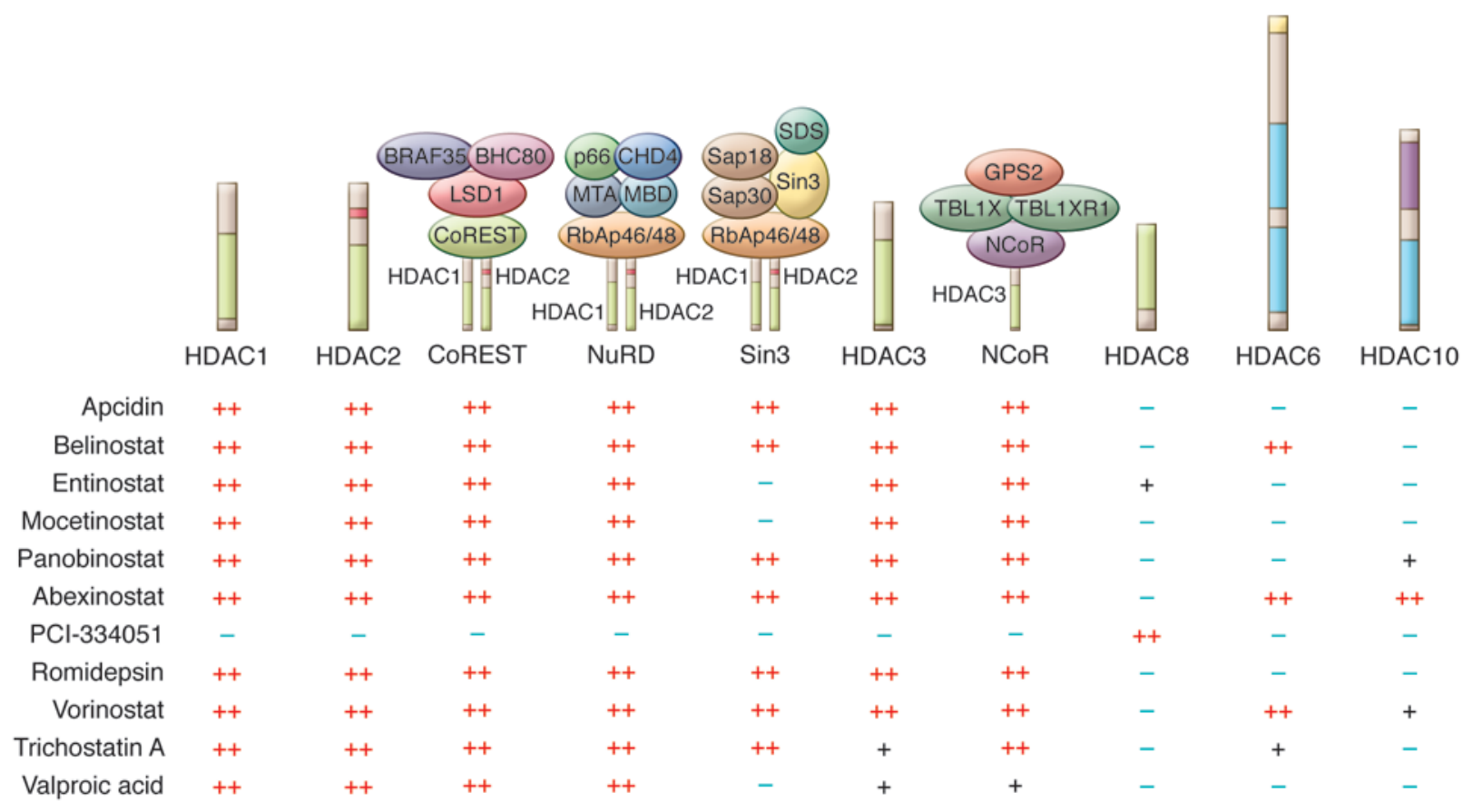

\section{Figure 2}

The specificity of HDACis for HDACs and associated protein complexes. The specificity of HDACis for HDAC isoforms has been recently reclassified using sophisticated chemoproteomics and chemical phylogenetic approaches, revealing that HDACis have surprising affinity for both HDACs and individual HDAC-dependent multiprotein complexes $(34,35)$. This new specificity is shown for class I HDAC1, $-2,-3$, and -8 as well as class IIb HDAC6 and -10; specificity is calculated from average $\mathrm{K}_{\mathrm{D}}^{\text {app }}$ values generated by Bantscheff et al. and Bradner et al. $(34,35)$. Illustration based on data from Deubzer et al. (94), Bolden et al. (40), Bantscheff et al. (34), and Bradner et al. (35). BHC80, BRAF35-HDAC complex protein; CHD4, chromodomain helicase DNA binding protein 4; CoREST, REST corepressor 1; LSD1, lysine-specific demethylase 1; MTA, metastasis-associated protein; MBD, methyl-CpG binding domain protein; NuRD, nucleosome remodeling and deacetylase; RbAp46, retinoblastoma-binding protein p46; Sap30, Sin3A-associated protein, 30 kDa; SDS, serine dehydratase; TBL1X, transducin $\beta$-like 1X-linked; TBL1XR1, transducin $\beta$-like 1 X-linked receptor 1; GPS2, G protein pathway suppressor 2 .

under investigation. While HDACis were historically identified on the basis of their ability to induce tumor cell differentiation (38, 39 ), induction of tumor cell apoptosis is the biological outcome most often reported (40). Indeed, using preclinical models, we and others have identified a direct link between HDACi-induced tumor cell apoptosis and therapeutic efficacy $(22,41-43)$. However, even here, divergent views exist regarding the importance of the intrinsic apoptotic pathway mediated by the interplay between proapoptotic and antiapoptotic Bcl-2 family proteins $(41,42,44)$, versus the extrinsic pathway mediated by death receptors (e.g., TRAIL receptors, Fas) and their cognate ligands (e.g., TRAIL, FasL) (21, 22). There is a link between altered gene expression and the induction of apoptosis, with histone hyperacetylation observed at promoters of apoptosis-inducing genes such as TNFSF10 (encoding TRAIL; ref. 22) and BMF (encoding the proapoptotic Bcl-2 family member Bmf; ref. 45), and changes in activity of transcription factors due to acetylation, such as inhibition of SP1 and C/EBP $\alpha$, leading to downregulation of the antiapoptotic protein $\mathrm{Bcl}-2$ (18), following HDACi treatment. It is therefore likely that both the threshold of apoptosis induction and the mechanism by which death can be triggered by HDACis is determined by the interaction between the oncogenic lesions and the intrinsic apoptosis-signaling pathways active within each cell.

Two reports have provided potential insights into biomarkers for response to HDACis. The first demonstrated that enhanced
JAK/STAT signaling negatively affected HDACi-induced death of CTCL cells, and constitutive accumulation of STAT1 in the nucleus and high levels of phosphorylated STAT3 correlated with a lack of response to vorinostat in clinical trials (46). The second approach incorporated a sophisticated loss-of-function genetic screen that identified human RAD23 homolog B (HR23B) as important for HDACi-induced apoptosis (47). Subsequent studies showed a correlation between HR23B expression and clinical response to vorinostat (48), and the interaction between Hsp90 and HDAC6 was identified as being the crucial functional effector mechanism delineating relative sensitivity to HDACi-induced apoptosis through regulated HR23B expression (49). Whether there is any functional interplay between JAK/STAT signaling and HR23B remains uncertain. Moreover, as HR23B expression can regulate sensitivity to HDACis that are very weak inhibitors of HDAC6 (e.g., apicidin, romidepsin) (48), it is unlikely that direct effects on the HDAC6/Hsp90 functional interaction account for the mechanistic role of HR23B in regulating HDACi-induced apoptosis.

How or why tumor cells are more sensitive to HDACi-induced apoptosis compared with matched, normal cells remains an intriguing and largely unanswered question. Previous studies indicated that tumor cells treated with HDACis preferentially accumulate ROS compared with treated normal cells, concomitant with enhanced expression of the reducing molecule thioredoxin 
in normal but not tumor cells (50). Recently, we utilized donormatched normal and transformed cells treated with vorinostat to identify a tumor cell-selective, proapoptotic gene expression signature containing effectors of the intrinsic apoptotic pathway that conferred tumor cell-selective apoptosis mediated by vorinostat and romidepsin (51). It is not clear why matched tumor and normal cells selectively regulate a subset of genes that confer tumor cell-selective, HDACi-mediated apoptosis. It is tempting to speculate that the cancer epigenome is altered in such a way as to predispose to altered expression of apoptotic genes in response to the transformation process, but definitive mechanistic evidence remains to be obtained.

\section{HDACis in the clinic}

The most successful clinical application of HDACis has been the use of vorinostat and romidepsin against refractory cutaneous and peripheral $\mathrm{T}$ cell lymphoma (52-55). In addition to these two FDA-approved agents, the butyrates, valproic acid, and newer compounds such as panobinostat (LBH589), givinostat (ITF2357), mocetinostat (MGCD01030), belinostat (PXD101), pracinostat (SB939), and entinostat (MS275) have been extensively studied in the clinic with varying results (56-58). Although some of these agents often demonstrate more potent antitumor effects than vorinostat and romidepsin in preclinical testing, none have thus far demonstrated novel mechanism(s) of action, vastly superior clinical activity, or more favorable toxicity profiles, and accordingly none have yet been registered for clinical use (56-58). While toxicities are largely manageable, in some instances cardiotoxicities as well as constitutional, hematologic, and gastrointestinal effects can be dose limiting (59). The efficacy of more than 20 different HDACis tested in the clinic has been largely restricted to hematological malignancies, with positive effects observed in Hodgkin lymphoma, multiple myeloma, and AML (some examples of these trials are listed in Table 2 and ref. 56). Despite positive responses in patients with hematologic malignancies, the effects of HDACis used as monotherapies against solid tumors has been disappointing, with few durable responses observed $(56,58)$. Current and future clinical studies using HDACis in combination with other agents, in particular with agents like bortezomib (velcade) in myeloma and other hematological malignancies, are ongoing $(56,57)$.

A second generation of orally available HDACis have been developed based on the chemical structure of clinically efficacious agents such as the hydroxamic acids (vorinostat) and the benzamides (entinostat, mocetinostat). Of these, several have entered the clinic including the class I-specific agents CHR-3966 (60), chidamide (CS055/HBI-8000) (61), class I- and class II-specific AR-42 (62), and hydroxamides quisinostat (JNJ-26481585) (63) and abexinostat (PCI- 24781) (64), and some preliminary results are described in Table 2. While still ongoing, early preclinical studies indicate these agents are more potent than the parental compounds, with improved pharmacodynamic and pharmacokinetic values, and are potentially less toxic. However, given that these new agents possess the same HDAC specificity profile (based on both chemoproteomic approaches [summarized in Figure 2] and traditional enzyme-based assays) as HDACis already in the clinic, it is unclear whether these newer agents will have improved clinical outcomes. The potency and reasonable toxicity profile of these agents suggests they may instead be useful in combinatorial therapeutic approaches.

\section{Novel and emerging HDACis in preclinical development}

Class I HDACis. HDAC1, -2 , and -3 are frequently overexpressed in human tumors, and knockdown of HDAC1 or -2 is sufficient to reduce tumor growth in vivo (see Table 1 for important tumor-specific examples). These studies provide a strong rationale for the development of class I- or isoform-specific inhibitors for the treatment of cancer. The recruitment of HDAC1-, HDAC2-, and/ or HDAC3-containing transcription regulatory complexes to leukemogenic fusion proteins indicates class I-specific agents may be particularly suited to the treatment of tumors driven by discrete oncogenic proteins. The pharmacologic development of class Ispecific or isoform-specific HDACis is currently underway, aided by clarification of the HDAC2 catalytic site following cocrystallization (65). Chemical modeling based on the HDAC2 crystal structure has allowed the generation of a series HDAC2-specific benzamides, and the inclusion of a sulfhydryl group may allow specific inhibition of HDAC1 $(65,66)$. Given the close sequence homology and observed functional redundancy mechanisms and compensatory expression relationships that occur between HDAC1 and -2, it will be of interest to determine whether these compounds confer different antitumor effects.

HDAC3 is emerging as a strong candidate for selective pharmacologic target inhibition in the oncology setting. The HDAC3-dependent NCoR complex can be recruited by the oncogenic fusion proteins PML-RAR $\alpha$, PLZF-RAR $\alpha$, or AML1-ETO in AML (19, 67). Furthermore, HDAC3 is required for the expression of almost half of the LPS-induced inflammatory genes in macrophages, and STAT1 levels were reduced in $H d a c 3^{-/-}$macrophages (68), suggesting a possible link. Conceivably, HDAC3 could act as the primary STAT1 and STAT3 deacetylase, which regulates the constitutive expression, deacetylation, and subsequent activation of STAT proteins in certain cancer types, a phenotype associated with resistance to HDACis (46). Other clues that suggest HDAC3 is an attractive target come from studies of Friedreich ataxia, a neurologic disorder caused by aberrant epigenetic silencing of the mutated frataxin (FXN) gene mediated by HDAC3. Treatment of mice bearing the Fxn mutation with an HDAC3-specific inhibitor, RG2833, resulted in decreased disease progression and enhanced survival, findings that led to the instigation of phase I trials (69, 70). It will be of interest to examine the efficacy of RG2833 in patients with cancer, especially in comparison to HDACis with high specificity for HDAC3 and NCoR, such as entinostat and mocetinostat (as represented in Figure 2 and ref. 34). Additionally, it will be important to determine whether patients develop immune-mediated complications, given the role of HDAC3 in inflammation (68). Moreover, the recent identification of putative tumor suppressor roles for HDAC1, -2, and -3 (25-27) raises some concerns about the clinical use of highly selective compounds that target these class I HDACs.

The role of HDAC8 in cancer is not as well established as other class I HDACs. HDAC8 has not been identified in any major corepressor complexes, and very few HDACis developed to date target HDAC8 with any potency (as depicted in Figure 2 and ref. 34). However, HDAC8 may have a particular role in solid tumors, as expression is enhanced in brain tumors, and knockdown revealed a role for maintaining the survival of lung, colon, and cervical cancer cells (Table 1 and refs. 50, 71). Thus, future studies are needed to determine whether inhibition of HDAC8 in solid tumors can enhance the traditionally suboptimal efficacy of HDACis in these malignancies (58). Solving the crystal structure of HDAC8 has 
assisted with in silico screening for appropriate chemical inhibitors $(50,72)$, the most promising of which is PCI-34051. Preclinical studies have suggested that PCI-34051 has activity against $\mathrm{T}$ cell malignancies, and while the mechanism is yet to be fully elucidated, HDAC 8 may be linked to PLC $\gamma 1$, a signal transducer specifically associated with the $\mathrm{T}$ cell receptor; together these proteins may induce apoptosis in T cell lymphomas (73). The development of PCI-34051 may allow for a better understanding of HDAC8 in cancer cells and can be utilized to identify any possible protein complexes containing HDAC8. It will be of interest to determine the potential effects of HDAC8 inhibition on normal T cells during treatment of solid malignancies. Assessment of PCI-34051 in $\mathrm{T}$ cell-mediated autoimmune diseases such as systemic lupus erythematosus (74) and colitis (75), in which pan-HDACis are currently undergoing investigation, may also be warranted.

Inhibitors of class II HDACs. The normal function of class IIa HDACs appears to be highly tissue specific (as indicated in Table 1), and there is a growing understanding that class IIa HDACs have weak deacetylase activity and may therefore more likely function as histone readers. Knockdown of HDAC4, -5, -7, and -9 induced the growth arrest of a variety of human solid tumor cell lines, and the depletion of HDAC4 alone was sufficient to induce apoptosis in colon and neuroblastoma tumors in vivo (Table 1), suggesting an important role for these HDACs in maintaining the survival of tumor cells $(76,77)$. Key to the non-catalytic activities of class IIa HDACs may be their capacity to act as scaffold proteins, incorporating enzymatically active HDACs into multiprotein complexes. For example, HDAC4, -5 , and -7 can recruit NCoR via recognition of the HDAC3 catalytic domain (78). Together these studies suggest there is a rationale for targeting class IIa HDACs as an anticancer approach. Ongoing research investigating the nonhistone protein targets of HDACs combined with the description of both HDAC4 (79) and HDAC7 (80) reader sites will likely allow for more detailed functional assessment of these proteins in cancer development. The recent discovery of trifluoromethyloxadiazole (TFMO), an agent with nanomolar potency against class II HDACs, will also enhance research in this area dramatically (81).

HDAC6 does not deacetylate histones, and accordingly, the anticancer effects of the HDAC6-specific inhibitors tubacin and rocilinostat (ACY-1215) are not associated with disrupted epigenetic control of gene transcription (82). A primary function of HDAC6 appears to be the regulation of protein degradation both via the aggresome (a structure that forms in response to misfolded proteins) and the regulation of Hsp90 chaperone activity. HDAC6 contains a ubiquitin-binding domain, unique among the HDACs, and tethers polyubiquitylated proteins to dynein motor for transport to the aggresome. Knockdown of HDAC6 led to the accumulation of polyubiquitylated, misfolded proteins due to lack of aggresome formation (83). HDAC6 also maintains activation of Hsp90 by deacetylation, and upon knockdown of HDAC6, Hsp90 becomes dissociated from its client proteins, leading to the ubiquitylation and degradation of immature, misfolded proteins (84). Several oncoproteins rely on Hsp90 for stability (e.g., Bcr-Abl, Her2); thus inhibition of HDAC6 can lead to their ubiquitin-mediated degradation, implicating HDAC6 as a promising anticancer target (85). However, inhibiting HDAC6 with tubacin was not sufficient to induce cell death (82), as protein aggregates can be degraded by the proteaseome. Indeed, the rational combination of tubacin or rocilinostat with the proteasome inhibitor bortezomib has been tested in preclinical models of multiple myeloma, with striking, synergistic anticancer effects observed $(86,87)$. The particular sensitivity of multiple myeloma to this combination therapy is thought to be due to the requirement of the malignant plasma cells to appropriately express and fold large amounts of immunoglobulin proteins; however, additional mechanisms are also likely to contribute (88). The combination of rocilinostat and the proteasome inhibitor bortezomib is currently under clinical investigation in multiple myeloma, and early results are promising (89). HDAC6 is responsible for $\alpha$-tubulin deacetylation (90), thus providing a sensitive biomarker for rocilinostat activity during clinical trials.

\section{Hybrid molecules targeting HDACs and other oncogenic proteins and pathways}

New medicinal chemistry approaches are being utilized to design more potent and specific inhibitors of class I HDACs. Compounds utilizing novel chemical scaffolds such as adamantane and noradamantane have been shown to inhibit HDACs at picomolar concentrations, and to inhibit tumor cell growth with limited toxicities in vivo at low nanomolar ranges (91). Chemical hybrid molecules containing both HDACi activity and an additional anticancer module are under development, and while the HDACi component is relatively broad spectrum, targeting HDACs concurrent with inhibition of PI3K (CUDC-907), tyrosine kinase (CUDC-101), or topoisomerase II (daunorubicin-vorinostat), or with $1 \alpha, 25$ vitamin D (triciferol) for nuclear receptor targeting (92), may be of interest for particular cancer subtypes. Additionally, an esterHDACi hybrid has been developed consisting of an HDACi and an esterase-sensitive chemical motif, which is specifically hydrolyzed by human monocytes and macrophages, trapping the active drug inside the cell (93). While this technology is currently useful for the inhibition of HDAC-regulated inflammatory cytokines and the treatment of inflammatory diseases, it also provides an impetus to utilize the hybrid technology to develop more cell type-specific HDACis for use in cancer.

\section{Conclusions}

The view that genetic lesions resulting in epigenetic deregulation is a major driver of tumor onset and progression is rapidly gaining acceptance by oncologists and cancer researchers. The development of HDACis as anticancer agents and the FDA approval of two agents, vorinostat and romidepsin, have provided the impetus to develop more potent HDACis and to target other epigenetic enzymes for oncology. While existing HDACis with somewhat broad target specificity have proven effective for the treatment of a relatively small population of patients with defined hematologic malignancies, their usefulness as single agents for the treatment of most other tumors has been marginal at best. Correlative data linking HDAC expression with patient prognosis and gene knockout as well as knockdown studies in preclinical mouse models of cancer are providing further clues as to the oncogenic and/ or tumor suppressor role of HDACs. Furthermore, these studies should lead to more sophisticated, evidenced-based development of HDACis with greater target specificity. Whether or not these agents will deliver significantly enhanced therapeutic efficacy and less toxicity as hypothesized remains to be determined.

\section{Acknowledgments}

R.W. Johnstone was supported by a National Health and Medical Research Council (NHMRC) Principal Research Fellowship and 
NHMRC program and project grants and by the Cancer Council Victoria (CCV), the Leukaemia Foundation of Australia, the Victorian Breast Cancer Research Consortium, and the Victorian Cancer Agency. A.C. West was supported by a CCV Fellowship.

1. Olsen CA. Expansion of the lysine acylation landscape. Angew Chem Int Ed Engl. 2012;51(16):3755-3756.

2. Tan MJ, et al. Identification of 67 histone marks and histone lysine crotonylation as a new type of histone modification. Cell. 2011;146(6):1015-1027.

3. Spange S, Wagner T, Heinzel T, Krämer OH. Acetylation of non-histone proteins modulates cellular signalling at multiple levels. Int J Biochem Cell Biol. 2009;41(1):185-198.

4. Fraga MF, et al. Loss of acetylation at Lys 16 and trimethylation at Lys20 of histone $\mathrm{H} 4$ is a common hallmark of human cancer. Nat Genet. 2005; 37(4):391-400.

5. Ozdag H, et al. Differential expression of selected histone modifier genes in human solid cancers. BMC Genomics. 2006;7:90.

6. Weichert W, et al. Histone deacetylases 1, 2 and 3 are highly expressed in prostate cancer and HDAC2 expression is associated with shorter PSA relapse time after radical prostatectomy. Br J Cancer. 2008; 98(3):604-610.

7. Weichert W, et al. Class I histone deacetylase expression has independent prognostic impact in human colorectal cancer: Specific role of class I histone deacetylases in vitro and in vivo. Clin Cancer Res. 2008;14(6):1669-1677.

8. Krusche CA, et al. Histone deacetylase-1 and-3 protein expression in human breast cancer: a tissue microarray analysis. Breast Cancer Res Treat. 2005; 90(1):15-23.

9. Minamiya Y, et al. Expression of histone deacetylase 1 correlates with a poor prognosis in patients with adenocarcinoma of the lung. Lung Cancer. 2011;74(2):300-304.

10. Osada H, Tatematsu Y, Saito H, Yatabe Y, Mitsudomi T, Takahashi T. Reduced expression of class II histone deacetylase genes is associated with poor prognosis in lung cancer patients. Int J Cancer. 2004;112(1):26-32.

11. Rikimaru $\mathrm{T}$, et al. Clinical significance of histone deacetylase 1 expression in patients with hepatocellular carcinoma. Oncology. 2007;72(1-2):69-74.

12. Weichert W, et al. Association of patterns of class I histone deacetylase expression with patient prognosis in gastric cancer: a retrospective analysis. Lancet Oncol. 2008;9(2):139-148.

13. Glozak MA, Seto E. Histone deacetylases and cancer. Oncogene. 2007;26(37):5420-5432.

14. Eot-Houllier G, Fulcrand G, Magnaghi-Jaulin L, Jaulin C. Histone deacetylase inhibitors and genomic instability. Cancer Lett. 2009;274(2):169-176.

15. Saji S, et al. Significance of HDAC6 regulation via estrogen signaling for cell motility and prognosis in estrogen receptor-positive breast cancer. Oncogene. 2005;24(28):4531-4539.

16. Marquard L, Gjerdrum LM, Christensen IJ, Jensen PB, Sehested M, Ralfkiaer E. Prognostic significance of the therapeutic targets histone deacetylase $1,2,6$ and acetylated histone H4 in cutaneous T-cell lymphoma. Histopathology. 2008;53(3):267-277.

17. Luo JY, Su F, Chen DL, Shiloh A, Gu W. Deacetylation of p 53 modulates its effect on cell growth and apoptosis. Nature. 2000;408(6810):377-381.

18. Duan H, Heckman CA, Boxer LM. Histone deacetylase inhibitors down-regulate bcl-2 expression and induce apoptosis in $\mathrm{t}(14 ; 18)$ lymphomas. Mol Cell Biol. 2005;25(5):1608-1619.

19. Gelmetti V, Zhang JS, Fanelli M, Minucci S, Pelicci PG, Lazar MA. Aberrant recruitment of the nuclear receptor corepressor-histone deacetylase complex by the acute myeloid leukemia fusion partner ETO. Mol Cell Biol. 1998;18(12):7185-7191.
20. Lin RJ, Nagy L, Inoue S, Shao WL, Miller WH, Evans RM. Role of the histone deacetylase complex in acute promyelocytic leukaemia. Nature. 1998;391(6669):811-814.

21. Insinga $A$, et al. Inhibitors of histone deacetylases induce tumor-selective apoptosis through activation of the death receptor pathway. Nat Med. 2004;11(1):71-76.

22. Nebbioso A, et al. Tumor-selective action of HDAC inhibitors involves TRAIL induction in acute myeloid leukemia cells. Nat Med. 2005;11(1):77-84.

23 . Taylor BS, et al. Frequent alterations and epigenetic silencing of differentiation pathway genes in structurally rearranged liposarcomas. Cancer Discov. 2011;1(7):587-597.

24. Ropero S, et al. A truncating mutation of HDAC2 in human cancers confers resistance to histone deacetylase inhibition. Nat Genet. 2006;38(5):566-569.

25. Bhaskara $S$, et al. Hdac 3 is essential for the maintenance of chromatin structure and genome stability. Cancer Cell. 2010;18(5):436-447.

26. Heideman MR, et al. Dosage-dependent tumor suppression by histone deacetylases 1 and 2 through regulation of c-Myc collaborating genes and p53 function. Blood. 2013;121(11):2038-2050.

27. Santoro F, et al. A dual role for Hdac1: oncosuppressor in tumorigenesis, oncogene in tumor maintenance. Blood. 2013;121(17):3459-3468.

28. Lin KT, Wang YW, Chen CT, Ho CM, Su WH, Jou YS. HDAC inhibitors augmented cell migration and metastasis through induction of PKCs leading to identification of low toxicity modalities for combination cancer therapy. Clin Cancer Res. 2012; 18(17):4691-4701.

29. Pulukuri SMK, Gorantla B, Rao JS. Inhibition of histone deacetylase activity promotes invasion of human cancer cells through activation of urokinase plasminogen activator. J Biol Chem. 2007; 282(49):35594-35603.

30. Vojinovic J, et al. Safety and efficacy of an oral histone deacetylase inhibitor in systemic-onset juvenile idiopathic arthritis. Arthritis Rheum. 2011; 63(5):1452-1458.

31. Bonfils C, et al. Evaluation of the pharmacodynamic effects of MGCD0103 from preclinical models to human using a novel HDAC enzyme assay. Clin Cancer Res. 2008;14(11):3441-3449.

32. Guenther MG, Barak O, Lazar MA. The SMRT and $\mathrm{N}-\mathrm{CoR}$ corepressors are activating cofactors for histone deacetylase 3. Mol Cell Biol. 2001; 21(18):6091-6101.

33. Zhang Y, Ng HH, Erdjument-Bromage H, Tempst P, Bird A, Reinberg D. Analysis of the NuRD subunits reveals a histone deacetylase core complex and a connection with DNA methylation. Genes Dev. 1999;13(15):1924-1935.

34. Bantscheff $\mathrm{M}$, et al. Chemoproteomics profiling of HDAC inhibitors reveals selective targeting of HDAC complexes. Nat Biotechnol. 2011;29(3):255-265.

35. Bradner JE, et al. Chemical phylogenetics of histone deacetylases. Nat Chem Biol. 2010;6(3):238-243.

36. Deardorff MA, et al. HDAC8 mutations in Cornelia de Lange syndrome affect the cohesin acetylation cycle. Nature. 2012;489(7415):313-317.

37. Scott GK, et al. Destabilization of ERBB2 transcripts by targeting 3'untranslated region messenger RNA associated HuR and histone deacetylase-6. Mol Cancer Res. 2008;6(7):1250-1258.

38. Leder A, Leder P. Butyric acid, a potent inducer of erythroid differentiation in cultured erythroleukemic cells. Cell. 1975;5(3):319-322.

39. Riggs MG, Whittaker RG, Neumann JR, Ingram VM.
n-Butyrate causes histone modification in HeLa and Friend erythroleukaemia cells. Nature. 1977; 268(5619):462-464.

40. Bolden JE, Peart MJ, Johnstone RW. Anticancer activities of histone deacetylase inhibitors. Nat Rev Drug Discov. 2006;5(9):769-784.

41. Ellis L, et al. The histone deacetylase inhibitors LAQ824 and LBH589 do not require death receptor signaling or a functional apoptosome to mediate tumor cell death or therapeutic efficacy. Blood. 2009;114(2):380-393.

42. Lindemann RK, et al. Analysis of the apoptotic and therapeutic activities of histone deacetylase inhibitors by using a mouse model of B cell lymphoma. Proc Natl Acad Sci U S A. 2007;104(19):8071-8076.

43. Newbold A, Lindemann RK, Cluse LA, Whitecross KF, Dear AE, Johnstone RW. Characterisation of the novel apoptotic and therapeutic activities of the histone deacetylase inhibitor romidepsin. Mol Cancer Ther. 2008;7(5):1066-1079.

44. Vrana JA, et al. Induction of apoptosis in U937 human leukemia cells by suberoylanilide hydroxamic acid (SAHA) proceeds through pathways that are regulated by Bcl-2/Bcl-XL, c-Jun, and p21CIP1, but independent of p53. Oncogene. 1999;18(50):7016-7025.

45. Zhang Y, Adachi M, Kawamura R, Imai K. Bmf is a possible mediator in histone deacetylase inhibitors FK228 and CBHA-induced apoptosis. Cell Death Differ. 2005;13(1):129-140.

46. Fantin VR, et al. Constitutive activation of signal transducers and activators of transcription predicts vorinostat resistance in cutaneous T-cell lymphoma. Cancer Res. 2008;68(10):3785-3794.

47. Fotheringham S, et al. Genome-wide loss-of-function screen reveals an important role for the proteasome in HDAC inhibitor-induced apoptosis. Cancer Cell. 2009;15(1):57-66.

48. Khan O, et al. HR23B is a biomarker for tumor sensitivity to HDAC inhibitor-based therapy. Proc Natl Acad Sci U S A. 2010;107(14):6532-6537.

49. New $M$, et al. A regulatory circuit that involves HR23B and HDAC6 governs the biological response to HDAC inhibitors. Cell Death Differ. 2013; 20(10):1306-1316.

50. Vannini A, et al. Crystal structure of a eukaryotic zinc-dependent histone deacetylase, human HDAC8, complexed with a hydroxamic acid inhibitor. Proc Natl Acad Sci U S A. 2004;101(42):15064-15069.

51. Bolden JE, et al. HDAC inhibitors induce tumorcell-selective pro-apoptotic transcriptional responses. Cell Death Dis. 2013;4:e519.

52. Duvic M, et al. Phase 2 trial of oral vorinostat (suberoylanilide hydroxamic acid, SAHA) for refractory cutaneous T-cell lymphoma (CTCL). Blood. 2007; 109(1):31-39

53. Olsen EA, et al. Phase IIb multicenter trial of vorinostat in patients with persistent, progressive, or treatment refractory cutaneous T-cell lymphoma. J Clin Oncol. 2007;25(21):3109-3115.

54. Whittaker SJ, et al. Final results from a multicenter, international, pivotal study of romidepsin in refractory cutaneous T-cell lymphoma. JClin Oncol. 2010; 28(29):4485-4491.

55. Piekarz RL, et al. Phase II multi-institutional trial of the histone deacetylase inhibitor romidepsin as monotherapy for patients with cutaneous T-cell lymphoma. J Clin Oncol. 2009;27(32):5410-5417.

56. Nebbioso A, Carafa V, Benedetti R, Altucci L. Trials with 'epigenetic' drugs: An update. Mol Oncol. 2012; 6(6):657-682.

57. New M, Olzscha H, La Thangue NB. HDAC inhibitor-based therapies: can we interpret the code? Mol 
Oncol. 2012;6(6):637-656.

58. Qiu TZ, et al. Effects of treatment with histone deacetylase inhibitors in solid tumors: a review based on 30 clinical trials. Future Oncol. 2013:9(2):255-269.

59. Gryder BE, Sodji QH, Oyelere AK. Targeted cancer therapy: giving histone deacetylase inhibitors all they need to succeed. Future Med Chem. 2012:4(4):505-524.

60. Banerji U, et al. A phase I pharmacokinetic and pharmacodynamic study of CHR-3996, an oral class selective histone deacetylase inhibitor in refractory solid tumors. Clin Cancer Res. 2012;18(9):2687-2694.

61. Dong M, et al. Phase I study of chidamide (CS055 HBI-8000), a new histone deacetylase inhibitor, in patients with advanced solid tumors and lymphomas. Cancer Chemother Pharmacol. 2012;69(6):1413-1422.

62. Hofmeister CC, et al. Phase I study of AR-42 in relapsed multiple myeloma and lymphoma. Presented at: 54th ASH Annual Meeting and Exposition; December 8-11, 2012; Atlanta, Georgia, USA https://ash.confex.com/ash/2012/webprogram/ Paper47235.html. Accessed November 15, 2013.

63. Child F, et al. Phase 2 Multicenter trial of oral quisinostat, a histone deacetylase inhibitor, in patients with previously treated stage IB-IVA cutaneous T-cell lymphoma. Presented at: 54th ASH Annual Meeting and Exposition; December 8-11, 2012; Atlanta, Georgia, USA. https://ash.confex. com/ash/2012/webprogram/Paper46292.html Accessed November 15, 2013

64. Evens AM, et al. A phase II multicenter study of the histone deacetylase inhibitor (HDACi) abexinostat (PCI-24781) in relapsed/refractory follicular lymphoma (FL) and mantle cell lymphoma (MCL). Presented at: 54th ASH Annual Meeting and Exposition; December 8-11, 2012; Atlanta, Georgia, USA https://ash.confex.com/ash/2012/webprogram/ Paper50038.html. Accessed November 15, 2013.

65. Bressi JC, et al. Exploration of the HDAC2 foot pocket: Synthesis and SAR of substituted $\mathrm{N}$-(2-aminophenyl)benzamides. Bioorg Med Chem Lett. 2010;20(10):3142-3145.

66. Zhang L, Li MY, Zhang LH, Xu WF. Discovering the binding modes of natural products with histone deacetylase 1. Med Chem. 2013;9(1):126-132.

67. Grignani F, et al. Fusion proteins of the retinoic acid receptor-alpha recruit histone deacetylase in promyelocytic leukaemia. Nature. 1998;391(6669):815-818

68. Chen XF, et al. Requirement for the histone deacetylase $\mathrm{Hdac} 3$ for the inflammatory gene expression program in macrophages. Proc Natl Acad Sci U S A. 2012;109(42):E2865-E2874.

69. Sandi C, et al. Prolonged treatment with pimelic o-aminobenzamide HDAC inhibitors ameliorates the disease phenotype of a Friedreich ataxia mouse model. Neurobiol Dis. 2011;42(3):496-505.

70. Soragni E, Xu CP, Plasterer HL, Jacques V, Rusche JR, Gottesfeld JM. Rationale for the development of 2-aminobenzamide histone deacetylase inhibitors as therapeutics for Friedreich ataxia. J Child Neurol. 2012;27(9):1164-1173.

71. Oehme I, et al. Histone deacetylase 8 in neuroblastoma tumorigenesis. Clin Cancer Res. 2009 15(1):91-99.

72. Somoza JR, et al. Structural snapshots of human HDAC8 provide insights into the class I histone deacetylases. Structure. 2004;12(7):1325-1334.

73. Balasubramanian S, Ramos J, Luo W, Sirisawad $\mathrm{M}$, Verner E, Buggy JJ. A novel histone deacetylase 8 (HDAC8)-specific inhibitor PCI-34051 induces apoptosis in T-cell lymphomas. Lenkemia. 2008; 22(5):1026-1034.

74. Reilly CM, et al. Modulation of renal disease in $\mathrm{MRL} / \mathrm{lpr}$ mice by suberoylanilide hydroxamic acid. J Immunol. 2004;173(6):4171-4178.

75. Tao R, et al. Deacetylase inhibition promotes the generation and function of regulatory T cells. Nat Med. 2007;13(11):1299-1307.

76. Wilson AJ, et al. HDAC4 promotes growth of colon cancer cells via repression of p21. Mol Biol Cell. 2008;19(10):4062-4075

77. Mottet D, et al. HDAC4 represses p21(WAF1/ Cip1) expression in human cancer cells through a Sp1-dependent, p53-independent mechanism. Oncogene. 2009;28(2):243-256.

78. Fischle W, et al. Enzymatic activity associated with class IIHDACs is dependent on a multiprotein complex containing HDAC 3 and SMRT/N-CoR Mol Cell. 2002;9(1):45-57.

79. Bottomley MJ, et al. Structural and functional analysis of the human HDAC4 catalytic domain reveals a regulatory structural zinc-binding domain. J Biol Chem. 2008;283(39):26694-26704.

80. Schuetz A, et al. Human HDAC7 harbors a class IIa histone deacetylase-specific zinc binding motif and cryptic deacetylase activity. J Biol Chem. 2008;283(17):11355-11363.

81. Lobera M, et al. Selective class IIa histone deacetylase inhibition via a nonchelating zinc-binding group. Nat Chem Biol. 2013;9(5):319-325.

82. Haggarty SJ, Koeller KM, Wong JC, Grozinger CM, Schreiber SL. Domain-selective small-molecule inhibitor of histone deacetylase 6 (HDAC6)-mediated tubulin deacetylation. Proc Natl Acad Sci U S A. 2003;100(8):4389-4394

83. Kawaguchi Y, Kovacs JJ, McLaurin A, Vance JM, Ito A, Yao TP. The deacetylase HDAC6 regulates aggresome formation and cell viability in response to misfolded protein stress. Cell. 2003;115(6):727-738.

84. Kovacs JJ, et al. HDAC6 regulates Hsp90 acetylation and chaperone-dependent activation of glucocorticoid receptor. Mol Cell. 2005;18(5):601-607.

85. Bali P, et al. Inhibition of histone deacetylase 6 acetylates and disrupts the chaperone function of heat shock protein $90-$ a novel basis for antileukemia activity of histone deacetylase inhibitors. J Biol Chem. 2005;280(29):26729-26734

86. Santo L, et al. Preclinical activity, pharmacodynamic, and pharmacokinetic properties of a selective HDAC6 inhibitor, ACY-1215, in combination with bortezomib in multiple myeloma. Blood. 2012; 119(11):2579-2589

87. Hideshima T, et al. Small-molecule inhibition of proteasome and aggresome function induces synergistic antitumor activity in multiple myeloma. Proc Natl Acad Sci U S A. 2005;102(24):8567-8572.

88. Hideshima T, Richardson PG, Anderson KC. Mechanism of action of proteasome inhibitors and deacetylase inhibitors and the biological basis of synergy in multiple myeloma. Mol Cancer Ther. 2011;10(11):2034-2042.

89. Raje N, et al. Rocilinostat (ACY-1215), a selective HDAC6 inhibitor, alone and in combination with bortezomib in multiple myeloma: Preliminary results from the first-in-humans phase I/II study. Presented at: 54th ASH Annual Meeting and Exposition; December 8-11, 2012; Atlanta, Georgia, USA. https://ash.confex.com/ash/2012/ webprogram/Paper52013.html. Accessed November 15, 2013.

90. Hubbert C, et al. HDAC6 is a microtubule-associated deacetylase. Nature. 2002;417(6887):455-458.

91. Gopalan B, et al. Discovery of adamantane based highly potent HDAC inhibitors. Bioorg Med Chem Lett. 2013;23(9):2532-2537.

92. Delcuve GP, Khan DH, Davie JR. Targeting class I histone deacetylases in cancer therapy. Expert Opin Ther Targets. 2013;17(1):29-41

93. Needham LA, et al. Drug targeting to monocytes and macrophages using esterase-sensitive chemical motifs. J Pharmacol Exp Ther. 2011;339(1):132-142.

94. Deubzer HE, et al. HDAC11 is a novel drug target in carcinomas. Int J Cancer. 2013;132(9):2200-2208.

95. Zhang ZH, et al. Quantitation of HDAC1 mRNA expression in invasive carcinoma of the breast. Breast Cancer Res Treat. 2005;94(1):11-16.

96. Adams H, Fritzsche FR, Dirnhofer S, Kristiansen G,
Tzankov A. Class I histone deacetylases 1, 2 and 3 are highly expressed in classical Hodgkin's lymphoma. Expert Opin Ther Targets. 2010;14(6):577-584.

97. Hiebert SW, et al. Mechanisms of transcriptional repression by the $\mathrm{t}(8 ; 21)-\mathrm{t}(12 ; 21)$-, and inv(16)encoded fusion proteins. Cancer Chemother Pharmacol. 2001;48(suppl 1):S31-S34.

98. David G, Alland L, Hong SH, Wong CW, DePinho RA, Dejean A. Histone deacetylase associated with $\mathrm{mSin} 3 \mathrm{~A}$ mediates repression by the acute promyelocytic leukemia-associated PLZF protein. Oncogene. 1998;16(19):2549-2556.

99. Dhordain P, et al. Corepressor SMRT binds the $\mathrm{BTB} / \mathrm{POZ}$ repressing domain of the LAZ3/ BCL6 oncoprotein. Proc Natl Acad Sci U S A. 1997; 94(20):10762-10767.

100. Wilson AJ, et al. Histone deacetylase 3 (HDAC3) and other class IHDACs regulate colon cell maturation and p21 expression and are deregulated in human colon cancer.J Biol Chem. 2006;281(19):13548-13558.

101.Yamaguchi T, et al. Histone deacetylases 1 and 2 act in concert to promote the G1-to-S progression. Genes Dev. 2010;24(5):455-469.

102.Santoro F, et al. A dual role for Hdac1: oncosuppressor in tumorigenesis, oncogene in tumor maintenance. Blood. 2013;121(17):3459-3468.

103. Haberland M, Johnson A, Mokalled MH, Montgomery RL, Olson EN. Genetic dissection of histone deacetylase requirement in tumor cells. Proc Natl Acad Sci U S A. 2009;106(19):7751-7755.

104. Senese $S$, et al. Role for histone deacetylase 1 in human tumor cell proliferation. Mol Cell Biol. 2007; 27(13):4784-4795.

105.Glozak MA, Sengupta N, Zhang X, Seto E. Acetylation and deacetylation of non-histone proteins. Gene. 2005;363:15-23.

106. Wilting RH, et al. Overlapping functions of Hdac1 and Hdac2 in cell cycle regulation and haematopoiesis. EMBO J. 2010;29(15):2586-2597.

107. Song J, et al. Increased expression of historic deacetylase 2 is found in human gastric cancer. Apmis. 2005;113(4):264-268.

108.Jung $\mathrm{KH}$, et al. HDAC2 overexpression confers oncogenic potential to human lung cancer cells by deregulating expression of apoptosis and cell cycle proteins. J Cell Biochem. 2012;113(6):2167-2177.

109. Harms KL, Chen XB. Histone deacetylase 2 modulates p53 transcriptional activities through regulation of p53-DNA binding activity. Cancer Res. 2007;67(7):3145-3152.

110. Moreno DA, et al. Differential expression of HDAC3, HDAC7 and HDAC9 is associated with prognosis and survival in childhood acute lymphoblastic leukaemia. Br J Haematol. 2010;150(6):665-673

111.Hong SH, David G, Wong CW, Dejean A, Privalsky ML. SMRT corepressor interacts with PLZF and with the PML-retinoic acid receptor $\alpha(\operatorname{RAR} \alpha)$ and PLZF-RAR $\alpha$ oncoproteins associated with acute promyelocytic leukemia. Proc Natl Acad Sci U S A. 1997;94(17):9028-9033.

112. Kramer $\mathrm{OH}$, et al. A phosphorylation-acetylation switch regulates STAT1 signaling. Genes Dev. 2009;23(2):223-235.

113. Atsumi A, Tomita A, Kiyoi H, Naoe T. Histone deacetylase 3 (HDAC3) is recruited to target promoters by PML-RAR $\alpha$ as a component of the $\mathrm{N}-\mathrm{CoR}$ co-repressor complex to repress transcription in vivo. Biochem Biophys Res Commun. 2006;345(4):1471-1480.

114.Sun XJ, Wei L, Chen Q, Terek RM. HDAC4 represses vascular endothelial growth factor expression in chondrosarcoma by modulating RUNX2 activity. J Biol Chem. 2009;284(33):21881-21890.

115. Milde T, et al. HDAC5 and HDAC9 in medulloblastoma: novel markers for risk stratification and role in tumor cell growth. Clin Cancer Res. 2010; 16(12):3240-3252.

116. Malik S, et al. Histone deacetylase 7 and FoxA1 in estrogen-mediated repression of RPRM. Mol Cell 
Biol. 2010;30(2):399-412.

117. Kotian S, Liyanarachchi S, Zelent A, Parvin JD. Histone deacetylases 9 and 10 are required for homologous recombination. J Biol Chem. 2011; 286(10):7722-7726.

118. Zhang ZH, et al. HDAC6 expression is correlated with better survival in breast cancer. Clin Cancer Res. 2004;10(20):6962-6968.

119. Park JH, et al. Class II histone deacetylases play pivotal roles in heat shock protein 90-mediated proteasomal degradation of vascular endothelial growth factor receptors. Biochem Biophys Res Commun. 2008;368(2):318-322.

120. Rhodes DR, et al. ONCOMINE: A cancer microarray database and integrated data-mining platform. Neoplasia. 2004;6(1):1-6.

121. Gao L, Cueto MA, Asselbergs F, Atadja P. Cloning and functional characterization of HDAC11, a novel member of the human histone deacetylase family. J Biol Chem. 2002;277(28):25748-25755.

122. Duvic M, et al. Panobinostat activity in both bexarotene-exposed and -naive patients with refractory cutaneous T-cell lymphoma: Results of a phase II trial. Eur J Cancer. 2013;49(2):386-394.

123. Ghobrial IM, et al. Final results of the phase II Trial of single agent panobinostat (LBH589) in relapsed or relapsed/refractory Waldenstrom macroglobulinemia. Paper presented at: 53rd ASH Annual Meeting and Exposition; December 10-13, 2011; San Diego, California, USA. https://ash.confex. com/ash/2011/webprogram/Paper39751.html. Accessed November 15, 2013.

124. Younes A, et al. Panobinostat in patients with relapsed/refractory Hodgkin's lymphoma after autologous stem-cell transplantation: results of a phase II study. J Clin Oncol. 2012;30(18):2197-2203.

125. Giaccone G, et al. Phase II study of belinostat in patients with recurrent or refractory advanced thymic epithelial tumors. J Clin Oncol. 2011; 29(15):2052-2059.

126. Gore L, et al. A phase I and pharmacokinetic study of the oral histone deacetylase inhibitor, MS-275, in patients with refractory solid tumors and lymphomas. Clin Cancer Res. 2008;14(14):4517-4525.

127. Hauschild A, et al. Multicenter phase II trial of the historic deacetylase inhibitor pyridylmethyl-N-\{4- (2-aminophenyl)-carbamoyl -benzyl\}-carbamate in pretreated metastatic melanoma. Melanoma Res. 2008;18(4):274-278.

128. Ryan QC, et al. Phase I and pharmacokinetic study of MS-275, a histone deacetylase inhibitor, in patients with advanced and refractory solid tumors or lymphoma. J Clin Oncol. 2005;23(17):3912-3922.

129. Younes A, et al. Mocetinostat for relapsed classical Hodgkin's lymphoma: an open-label, single-arm, phase 2 trial. Lancet Oncol. 2011;12(13):1222-1228.

130. Rambaldi A, et al. A pilot study of the HistoneDeacetylase inhibitor Givinostat in patients with JAK2V617F positive chronic myeloproliferative neoplasms. BrJ Haematol. 2010;150(4):446-455.

131. Razak ARA, et al. Phase I clinical, pharmacokinetic and pharmacodynamic study of SB939, an oral histone deacetylase (HDAC) inhibitor, in patients with advanced solid tumours. Br J Cancer. 2011; 104(5):756-762.

132. Yong WP, et al. Phase I and pharmacodynamic study of an orally administered novel inhibitor of histone deacetylases, SB939, in patients with refractory solid malignancies. Ann Oncol.2011;22(11):2516-2522. 